\title{
Metabolic and Pancreatic Derangement in Type 2 Diabetic Rat
}

\author{
Liza $\mathbf{N}^{1}$, Nor Asiah $\mathrm{MN}^{2,3}$, Nor Hidayah $\mathrm{AB}^{4}$. Wan Amir Nizam $\mathrm{WA}^{2}$ \\ ${ }^{1}$ Department of Physiology, School of Medical Sciences, Universiti Sains Malaysia, Health Campus, Kelantan, Malaysia. \\ ${ }^{2}$ Biomedicine Programme, School of Health Sciences, Universiti Sains Malaysia, Health Campus, Kelantan, Malaysia. \\ ${ }^{3}$ Faculty of Health Science, Universiti Sultan Zainal Abidin, Gong Badak Campus, Kuala Nerus, Terengganu, Malaysia. \\ ${ }^{4}$ Faculty of Medicine, Universiti Sultan Zainal Abidin, Medical Campus, Kuala Terengganu, Terengganu, Malaysia.
}

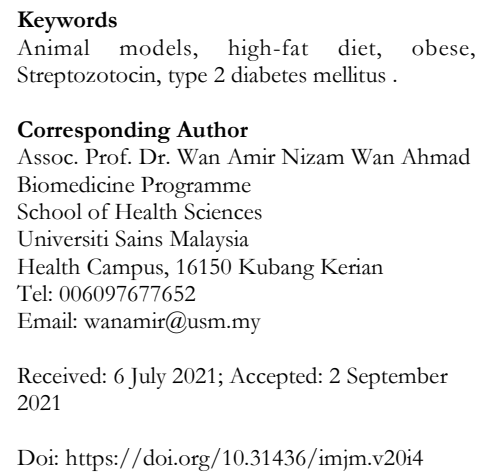

\begin{abstract}
INTRODUCTION: The prevalence of diabetes mellitus is increasing globally, despite the best current therapies available. A large diversity of animal models has been developed for a better understanding of the pathogenesis of diabetes mellitus. This study aimed to develop a rat model that mimics the metabolic characteristics of human type 2 diabetes mellitus. MATERIALS AND METHODS: : Twenty-four male Sprague-Dawley rats (200$250 \mathrm{~g}$ ) were divided into two groups and fed either a commercially available standard pellet diet $(n=8)$ or a self-prepared high-fat diet (HFD) $(n=16)$ for 6 weeks. HFD-fed rats were significantly obese compared to standard-fed rats. Eight of the obese rats were injected a single low dose of streptozotocin (STZ) at $35 \mathrm{mg} / \mathrm{kg}$ intraperitoneally to induce type 2 diabetic rat (T2DR) and were monitored for 6 weeks. RESULTS: The weekly fasting blood glucose levels (FBG) in T2DR remained consistently high throughout the 6 weeks. The T2DR group exhibits decreased bodyweight, increased water intake, increased 24-hour urine volume with microalbuminuria, hyperlipidaemia, altered liver and kidney functions, and structural changes of the islet of Langerhans. CONCLUSIONS: This model simulates the natural disease progression and metabolic characteristics of type 2 diabetic patients. This model mimics the human syndrome that can be maintained at a reasonable cost and is easy to develop.
\end{abstract}

\section{INTRODUCTION}

Diabetes mellitus, a heterogeneous metabolic disorder due to impaired insulin secretion, defective insulin action, or both that leads to hyperglycaemia. ${ }^{1}$ The prevalence of this disease has increased worldwide and the disease is present in every population in all regions that have become one of the medical challenges. International Diabetes Federation (IDF) reported that about 415 million adults had DM with the age range is between 20 and 79 years in 2015 and is expected to increase to another 200 million by $2040 .^{2} \mathrm{DM}$ is classified into four types; type 1 diabetes (T1DM), Type 2 diabetes (T2DM), gestational diabetes mellitus (GDM), and specific types such as pancreatitis and drug-induced diabetes. ${ }^{3}$ In chronic conditions, DM causes damage to various organ systems that may develop life-threatening health complications or mortality risk especially when it involves microvascular systems, including retinopathy, nephropathy, and neuropathy.

Among the cases, T2DM accounts for around 90\% of diabetes, especially those older than 45 years or middle-aged adults. The association of obesity with T2DM has been recognised for decades. ${ }^{4}$ Overweight or obesity causes some degree of insulin resistance, so most but not all patients with T2DM are overweight or obese. ${ }^{3}$ Increasing age, obesity, and lack of physical activity increase the risk of developing T2DM. ${ }^{5}$ In this modern society, the increasing prevalence of obesity and changing of lifestyle causes many children, adolescents, and younger adults to suffer T2DM. ${ }^{6}$ In T2DM, there is either insulin resistance, diminished response to insulin, or the beta cell is unable to sufficiently compensate. At the earlier stage, the glucose levels are in the normal range due to a compensatory increase in insulin secretion. However, the beta cells change as the disease progresses, and the secretion of insulin is unable to maintain glucose homeostasis and subsequently causes hyperglycaemia.

Various animal models have been developed recently for a better understanding of the pathogenesis of diabetes mellitus. These animal models can be categorised into spontaneous or genetically derived diabetic animals, chemically induced diabetic, surgical diabetic animals, and diet-induced diabetic. ${ }^{7}$ 
Animal models are often preferable to experimental disease research due to ease of manipulation and supply. Besides, the conditions associated with the experiment must be closely controlled to obtain scientifically valid research. ${ }^{8}$ Although certain genetic models such as Zucker diabetic fatty (ZDF) rat and $\mathrm{db} / \mathrm{db}$ mouse are resembling human $\mathrm{T} 2 \mathrm{DM}$, these animals are expensive, difficult to maintain, and are not easily available. ${ }^{5}$ Obesity is closely linked to the development of T2DM in humans. Therefore, the majority of animal models of T2DM are obese with insulin resistance and/or beta-cell failure. Obesity can be induced by naturally occurring mutations, genetic manipulation, or high-fat feeding. Among pre-clinical animal models, rodents are the most widely used by many researchers to study metabolic disorders as they closely resemble human physiology, despite the pancreatic islet architecture differs from that of humans. ${ }^{10} \mathrm{~A}$ high-fat diet may cause insufficient compensation by the islets, which may lead to obesity, hyperinsulinaemia, and altered glucose homeostasis. ${ }^{11}$

Published evidence has shown that animal models play an important role in understanding the pathogenesis of diabetes and its complications. ${ }^{12,13}$ Besides, these models are important for the investigation and development of novel drugs in diabetes treatment. ${ }^{14}$ Numerous T2DM animal models have been used previously, ${ }^{15-18}$ which include insulin resistance models and/or beta-cell failure models. The ideal animal model of diabetes resembles the anatomy and pathophysiology of humans and has the potential to be utilised in clinical and pharmaceutical research that can be extrapolated to humans. Furthermore, a good model should be easy to develop and handle at a reasonable cost.

Alloxan (ALX) and streptozotocin (STZ) are the most potent diabetogenic chemicals that used in the research of diabetes. STZ is preferable to induce T1DM and T2DM diabetes because ALX is known to cause toxicity to the kidney as it has a narrow range of effective doses. ${ }^{19}$ The current study was initiated to develop an appropriate animal model which is analogous to human T2DM through a combination of a self-made high-fat diet and a low-dose STZ injection. This model closely mimics the natural history of the progress of diabetes from insulin resistance to beta-cell dysfunction.

\section{MATERIAL AND METHODS}

\section{Materials and reagents}

STZ was purchased from Sigma Aldrich, Germany. Ghee oil was purchased from Crispo, Crispo-Tato (M) Sdn Bhd, Kuala Lumpur, Malaysia. Calcium and vitamin D were purchased from Eurobio Sdn. Bhd. (Victoria, Australia).
Sodium pentobarbitone was purchased from Alfasan Woerden. Other chemicals were of the highest analytical grade available from local suppliers.

\section{Proximate analysis of standard pellet and high-fat diet}

The standard rat pellet, Altromin pellet was imported from Germany by Sterling Ascent, Malaysia. The high-fat diet (HFD) preparation was modified from the previous study, which included a combination of $68 \mathrm{~g}$ of standard rat pellet, $32 \mathrm{~g}$ of ghee oil, $300 \mathrm{mg}$ of calcium, and $100 \mathrm{IU}$ of vitamin D3 per $100 \mathrm{~g}$ of standard rat pellet. ${ }^{20}$ All the ingredients were thoroughly mixed and kept in the refrigerator at $4^{\circ} \mathrm{C}$ to make the mixture firm before giving it to the respective group. Nutrition compositions are shown in Table 1.

Table 1. Standard and high-fat diet nutritional compositions.

\begin{tabular}{lcc}
\hline Compositions (\%) & $\begin{array}{c}\text { Standard Diet } \\
(\mathbf{g} / \mathbf{1 0 0} \mathbf{~ g})\end{array}$ & $\begin{array}{c}\text { High-fat Diet (HFD) } \\
(\mathbf{g} / \mathbf{1 0 0} \mathbf{~ g})\end{array}$ \\
\hline Protein & 19.2 & 15.1 \\
Fibre & 6.1 & 18.7 \\
Fat & 4.1 & 31.1 \\
Moisture & 11.3 & 7.0 \\
Ash & 6.9 & 5.2 \\
Carbohydrate & 40.8 & 41.6 \\
Energy $(\mathrm{kcal} / 100 \mathrm{~g})$ & 319.8 & 507.0 \\
\hline
\end{tabular}

\section{Experimental design}

Twenty-four male Sprague-Dawley rats aged 8-10 weeks weighing 200-250 g were included in this study. Rats were procured from the Animal Research and Service Centre (ARASC), Universiti Sains Malaysia (USM), Malaysia. All procedures involving animal work have been carried out under the approval of the Institutional Animal Care and Use Committee (IACUC), USM (Ref: USM/ISCUC/2017/9110 (886)). The animals were individually acclimatised upon arrival for a week in a standard polypropylene cage, provided with a standard rat pellet and drinking water ad libitum. The animal room was set at a controlled temperature of $23 \pm 1{ }^{\circ} \mathrm{C}$ with a 12-hour light-dark cycle.

\section{Induction of Obesity}

Rats were divided into two groups and fed for 6 weeks either with standard rat pellet (Normal rat, $n=8$ ) or highfat diet (HFD, $n=16$ ) to induce obesity. The body weight of each rat was weighed weekly using a digital balance scale (A\&D HV-60KGL, Columbia). The difference in weight was calculated according to the difference between the initial and final body weight. Obesity was considered when the body mass index (BMI) was greater than $0.68 \mathrm{~g} / \mathrm{cm}^{2} .{ }^{21}$ The BMI was calculated using the following formula: body weight (g)/ length ${ }^{2}\left(\mathrm{~cm}^{2}\right)$. 
Eight of the obese rats $(n=8)$ were then subjected to Type 2 diabetes mellitus (T2DM) induction, assigned as Type 2 Diabetic Rat (T2DR). Rats were injected a single low dose of streptozotocin (STZ) at $35 \mathrm{mg} / \mathrm{kg}$ intraperitoneally.22 One week post-STZ induction, FBG was measured, and rats with FBG levels greater than $11.1 \mathrm{mmol} / \mathrm{L}$ (200 mg/dL) were considered diabetic. ${ }^{23}$ The experimental timeline for induction of diabetes mellitus is shown in Figure 1.

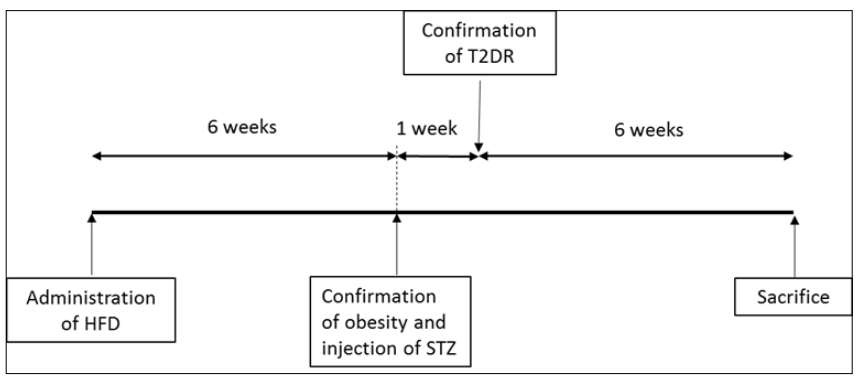

Figure 1. Experimental timeline for the induction of diabetes mellitus in rats. HFD: High-fat diet, T2DR: Type 2 diabetic rat, STZ: Streptozotocin.

\section{Measurement of Physical Parameters, 24-hour Urine Volume, and Microalbuminuria}

Rats were observed for another 6 weeks in each group. Physical parameters, including daily intake of food, daily intake of water, and body weight gain, have been measured. Rats were placed individually in a metabolic cage for the collection of urine for 24 hours (24-hour urine volume). Samples were sent to B.P. Clinical Lab Sdn. Bhd., Kota Bharu, Kelantan, Malaysia to measure the level of microalbuminuria using the immunoturbidimetric method (Architect, AbbottCi8200).

\section{Measurement of Fasting Blood Glucose and Insulin Levels}

The FBG was measured biweekly using the tail-pricks method in the dorsal vein using the portable Accu-Check Advantage glucometer (One Touch Ultra). Insulin levels in the plasma were determined by an Enzyme-Linked Immunosorbent Assay (ELISA) kit specific to rat insulin (Elabscience). The procedures were followed the manufacturer's protocols.

\section{Measurement of biochemical parameters}

Blood samples for the measurement of renal parameters, liver function tests, and lipid profiles were sent to B.P. Clinical Lab Sdn. Bhd., Kota Bharu, Kelantan, Malaysia. The atherogenic index of plasma (AIP) was calculated as a logarithmic transformation of the ratio of TG to HDL-C. ${ }^{24}$
Rats of each group were sacrificed with sodium pentobarbitone $(100 \mathrm{mg} / \mathrm{kg})$ via intraperitoneal injection at the end of the experiment. Blood samples, about 5-10 ml were collected via cardiac puncture into an EDTA-containing tube. Meanwhile, the pancreas was carefully isolated, excised, rinsed in ice-cold saline, and stored in 10\% neutral buffered formalin for 72 hours at room temperature for histological study.

\section{Histological Study of Pancreas}

The paraffin-embedded pancreas was sectioned at $3 \mu \mathrm{m}$ using a rotary microtome (Leica Biosystems). The tissue sections were then prepared on the glass slides and placed on a hot plate (HI1220; Leica Microsystems). The tissue sections were then deparaffinized using xylene and rehydrated by immersing in a series of decreasing concentrations of ethanol $(100 \%, 90 \%$, and $70 \%)$. The sections later were stained with hematoxylin and eosin ( $\mathrm{H} \& \mathrm{E})$, dehydrated by immersing in a series of increasing concentrations of ethanol followed by xylene. Following that, the slides were mounted and coverslipped. Slides were examined under a light microscope and the images were captured.

\section{Statistical Analysis}

Data were analyzed using Graph Pad Prism software version 9 for Windows (GraphPad, San Diego, CA). The results were expressed in mean (Standard Deviation; SD). The statistical differences between the means were determined using oneway analysis of variance (ANOVA) followed by the Tukey method for post-hoc analysis. All tests were two-tailed and a $p$-value of less than 0.05 was considered statistically significant.

\section{RESULTS}

\section{Physical parameters and fasting blood glucose between the standard diet and high-fat diet}

Physical parameters, including food intake, body weight, and body mass index (BMI) have been measured, as shown in Table 2. There was no significant difference in the food intake between the groups. The final body weight and BMI increased significantly in HFD compared to the standard diet. After six weeks of HFD, it was noticeable that rats had successfully achieved obesity levels, with BMI was greater than $0.68 \mathrm{~g} / \mathrm{cm}^{2}$. Besides, fasting blood glucose (FBG) also increased significantly in HFD compared to the standard diet but was within the normal range $(<11.1 \mathrm{mmol} / \mathrm{L})$. 
Table 2. Physical parameters and fasting blood glucose at 6 weeks

\begin{tabular}{lcc}
\hline & Standard Diet & $\begin{array}{c}\text { High-fat Diet } \\
\text { (HFD) }\end{array}$ \\
\hline Food intake (g/day) & $16.49(1.62)$ & $17.40(2.15)$ \\
Initial body weight $(\mathrm{g})$ & $211.90(11.55)$ & $218.70(11.86)$ \\
Final body weight $(\mathrm{g})$ & $282.40(15.73)$ & $451.00(30.58)^{\mathrm{a}}$ \\
Weight difference $(\mathrm{g})$ & $70.57(24.81)$ & $232.30(38.11)^{\mathrm{a}}$ \\
BMI g/cm & $0.55(0.02)$ & $0.71(0.03)^{\mathrm{a}}$ \\
FBG $(\mathrm{mmol} / \mathrm{L})$ & $4.88(0.43)$ & $5.57(0.30)^{\mathrm{a}}$
\end{tabular}

Data represent the mean (standard deviation), $\mathrm{n}=8$ (standard diet), $\mathrm{n}=16$ (high fat diet). a $p<0.0001$ compared to the standard diet. BMI: body mass index, FBG: Fasting blood glucose.

\section{Type 2 diabetes induction}

Eight obese rats $(n=8)$ were induced with a low dose of 35 $\mathrm{mg} / \mathrm{kg}$ streptozotocin (STZ) to become diabetic (Type 2 diabetic rat or T2DR). One week post-STZ induction, FBG, and insulin levels have been measured as shown in Figure 2. FBG was significantly higher in the T2DR group compared to Normal and Non-diabetic Obese rats (OB). There were, however, no significant differences in insulin levels between the groups. Besides, FBG in the T2DR group remains significantly higher throughout 6 weeks period of the study as shown in Figure 3.

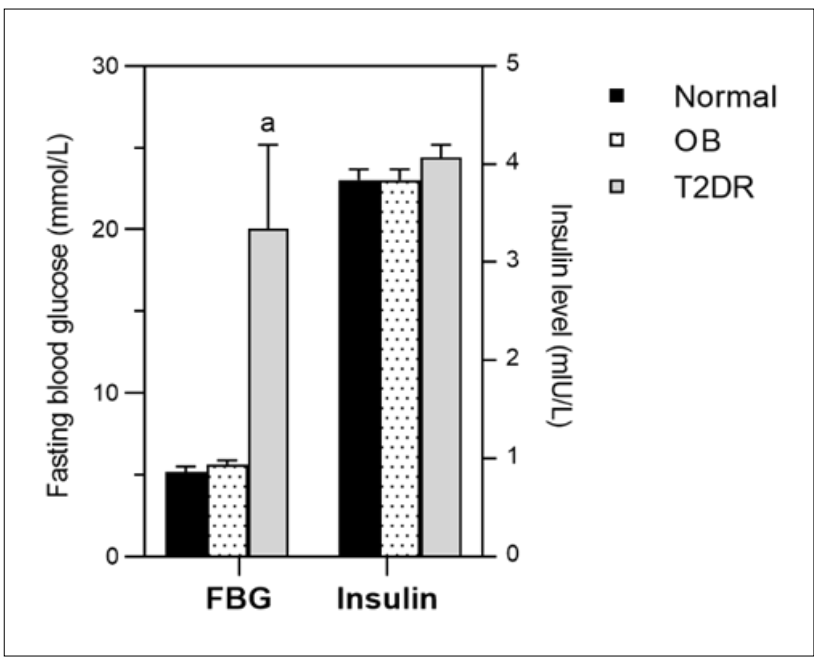

Figure 2. Levels of fasting blood glucose and insulin one week post Streptozotocin induction in Normal, Obese and Type 2 diabetic rats. ${ }^{a} p<0.001$ compared to Normal and Obese rats, $(n=8)$. OB: Obese rat, T2DR: Type 2 Diabetic rat.

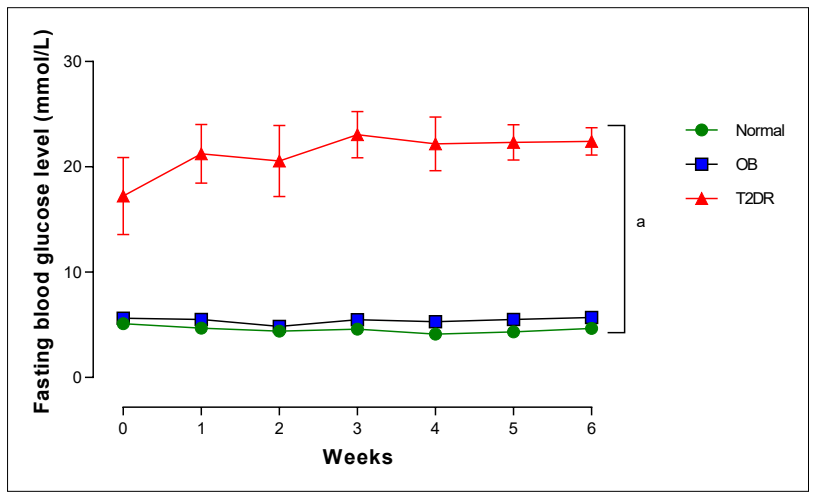

Figure 3. Levels of fasting blood glucose in Normal, Obese, and Type 2 diabetic rats. ( ${ }^{a} p<0.001$; main effects) $(n=8)$. OB: Obese rat, T2DR: Type 2 Diabetic rat.
Effects of diabetes mellitus on physical parameters, fasting blood glucose, 24-hour urine volume, and microalbuminuria

At the end of the study, in the OB group, there was a significant increase in food intake compared to the Normal group. The body weight gain was significantly decreased in the T2DR group compared to other groups. Meanwhile, water intake, 24-hour urine volume, and microalbuminuria increased significantly in the T2DR group compared to other groups as shown in Table 3.

Table 3 Physical parameters, fasting blood glucose, 24-hour urine volume, and microalbuminuria.

\begin{tabular}{|c|c|c|c|}
\hline & Normal & OB & T2DR \\
\hline $\begin{array}{l}\text { Food intake } \\
\text { (g/day) }\end{array}$ & $14.96(1.24)$ & $18.02(1.81)^{\mathrm{b}}$ & $17.14(1.17)$ \\
\hline Water intake (ml) & $35.00(4.00)$ & $33.81(4.94)$ & $79.37(3.60)^{\mathrm{a}}$ \\
\hline $\begin{array}{l}\text { Body weight gain } \\
\text { (g) }\end{array}$ & $61.60(5.98)$ & $119.1(36.52)$ & $-61.14(17.19)^{a}$ \\
\hline $\begin{array}{l}\text { FBG }(\mathrm{mmol} / \mathrm{L}) \\
\text { 24-h Urine } \\
\text { volume }(\mathrm{ml})\end{array}$ & $\begin{array}{l}4.55(0.31) \\
4.40(0.89)\end{array}$ & $\begin{array}{l}5.42(0.28) \\
4.50(1.00)\end{array}$ & $\begin{array}{l}21.29(1.97)^{\mathrm{a}} \\
44.17(11.33)^{\mathrm{a}}\end{array}$ \\
\hline $\begin{array}{l}\text { Urine microalbu- } \\
\text { minuria }(\mathrm{mg} / \mathrm{L})\end{array}$ & $19.75(12.84)$ & $25.00(7.45)$ & $46.20(14.87)^{\mathrm{a}}$ \\
\hline
\end{tabular}

Data represent the mean (standard deviation), $\mathrm{n}=8$ per group. ${ }^{\mathrm{a}} \mathrm{p}<0.05$ compared to Normal and Obese rats, $\mathrm{b} p<0.05$ compared to Normal rats. OB: Obese rat, T2DR: Type 2 Diabetic rat.

\section{Effects of diabetes mellitus on renal and liver parameters}

The levels of renal and liver parameters are shown in Table 4. In renal parameters, blood urea nitrogen (BUN) and creatinine were significantly increased in the T2DR group compared to Normal and OB groups. All the liver enzymes; aspartate transaminase (AST), alanine aminotransferase (ALT), and alkaline phosphatase (ALP) were significantly increased in the T2DR group compared to the Normal and OB groups. Also, total cholesterol, triglyceride, HDL-C, and atherogenic index (AI) were significantly increased in the T2DR group compared to Normal and OB groups.

\section{Effects of diabetes mellitus on histological changes of pancreas}

The histology of the pancreas was evaluated by hematoxylin and eosin (H\&E) staining as shown in Figure 4. The islet of Langerhans of the pancreatic tissues from the Normal group appeared as a cluster of pale staining cells, surrounded by more intensely staining pancreatic acini cells (Figure 4a). The round or elongated appearance of the islet of Langerhans is evenly scattered throughout the acini, and the homogenous 


\begin{tabular}{|c|c|c|c|}
\hline & Normal & OB & T2DR \\
\hline \multicolumn{4}{|l|}{ Renal parameters } \\
\hline BUN (mmol/L) & $7.11(0.65)$ & $6.46(0.70)$ & $9.61(1.11)^{\mathrm{a}}$ \\
\hline Creatinine $(\mu \mathrm{mol} / \mathrm{L})$ & $47.03(4.03)$ & $52.25(5.29)$ & $69.07(3.81)^{\mathrm{a}}$ \\
\hline Uric acid $(\mu \mathrm{mol} / \mathrm{L})$ & $133.80(10.22)$ & $140.86(7.54)$ & $158.40(6.12)$ \\
\hline Sodium (mmol/L) & $142.30(0.50)$ & $140.50(0.98)$ & $136.40(0.50)$ \\
\hline Potassium (mmol/L) & $6.830(0.27)$ & $7.78(0.26)$ & $7.50(0.25)$ \\
\hline \multicolumn{4}{|l|}{ Liver parameters } \\
\hline Total protein (g/L) & $66.89(3.40)$ & $73.00(8.02)$ & $67.75(2.56)$ \\
\hline Albumin $(g / L)$ & $27.44(1.67)$ & $29.75(1.99)$ & $27.13(0.90)$ \\
\hline Globulin (g/L) & $39.44(5.03)$ & $43.25(6.50)$ & $40.63(8.86)$ \\
\hline $\mathrm{A} / \mathrm{G}$ ratio $(\mathrm{g} / \mathrm{L})$ & $0.70(0.09)$ & $0.70(0.08)$ & $0.69(0.12)$ \\
\hline Total bilirubin $(\mu \mathrm{mol} / \mathrm{L})$ & $1.70(0.01)$ & $1.71(0.01)$ & $1.70(0.01)$ \\
\hline $\operatorname{AST}(\mathrm{U} / \mathrm{L})$ & $107.50(9.07)$ & $140.00(23.07)$ & $162.60(57.14)^{\mathrm{a}}$ \\
\hline $\operatorname{ALT}(\mathrm{U} / \mathrm{L})$ & $68.56(9.06)$ & $70.63(20.20)$ & $108.60(35.79)^{\mathrm{a}}$ \\
\hline $\operatorname{ALP}(\mathrm{U} / \mathrm{L})$ & $269.00(44.86)$ & $454.50(96.78)$ & $1459.00(178.3)^{\mathrm{a}}$ \\
\hline \multicolumn{4}{|l|}{ Lipid profiles } \\
\hline Total cholesterol (mmol/L) & $1.60(0.12)$ & $2.10(0.24)$ & $2.88(0.40)^{a}$ \\
\hline Triglycerides (mmol/L) & $1.17(0.45)$ & $2.32(0.81)$ & $4.90(1.78)^{\mathrm{a}}$ \\
\hline $\mathrm{LDL}(\mathrm{mmol} / \mathrm{L})$ & $0.58(0.25)$ & $1.60(1.28)$ & $3.68(1.64$ a \\
\hline HDL (mmol/L) & $0.44(0.05)$ & $0.60(0.22)$ & $0.60(0.07)$ \\
\hline AIP & $0.43(0.16)$ & $0.62(0.30)$ & $0.90(0.20)^{\mathrm{b}}$ \\
\hline
\end{tabular}

Data are presented as means (standard deviation), $\mathrm{n}=8$ per group. a $p<0.05$, b $p<0.01$ compared to Normal and Obese rats. OB: Obese rat, T2DR: Type 2 Diabetic rat, AIP: Atherogenic Index of Plasma

size of islet cells has been identified. Furthermore, the acinar cells were pyramidal-shaped with basal nuclei and apical acidophilic cytoplasm. In the OB group, the pancreatic islets were small in size with the presence of scattered fat deposits throughout the pancreatic acini (Figure 4b). However, the T2DR group demonstrated pathological changes of both exocrine and endocrine components as shown in Figure 4c. The islet of Langerhans appeared small in size, irregular in shape with the presence of scattered vacuolated cells inside. The acinar cells appeared swollen with the presence of fat infiltrations that are scattered within the lobules.
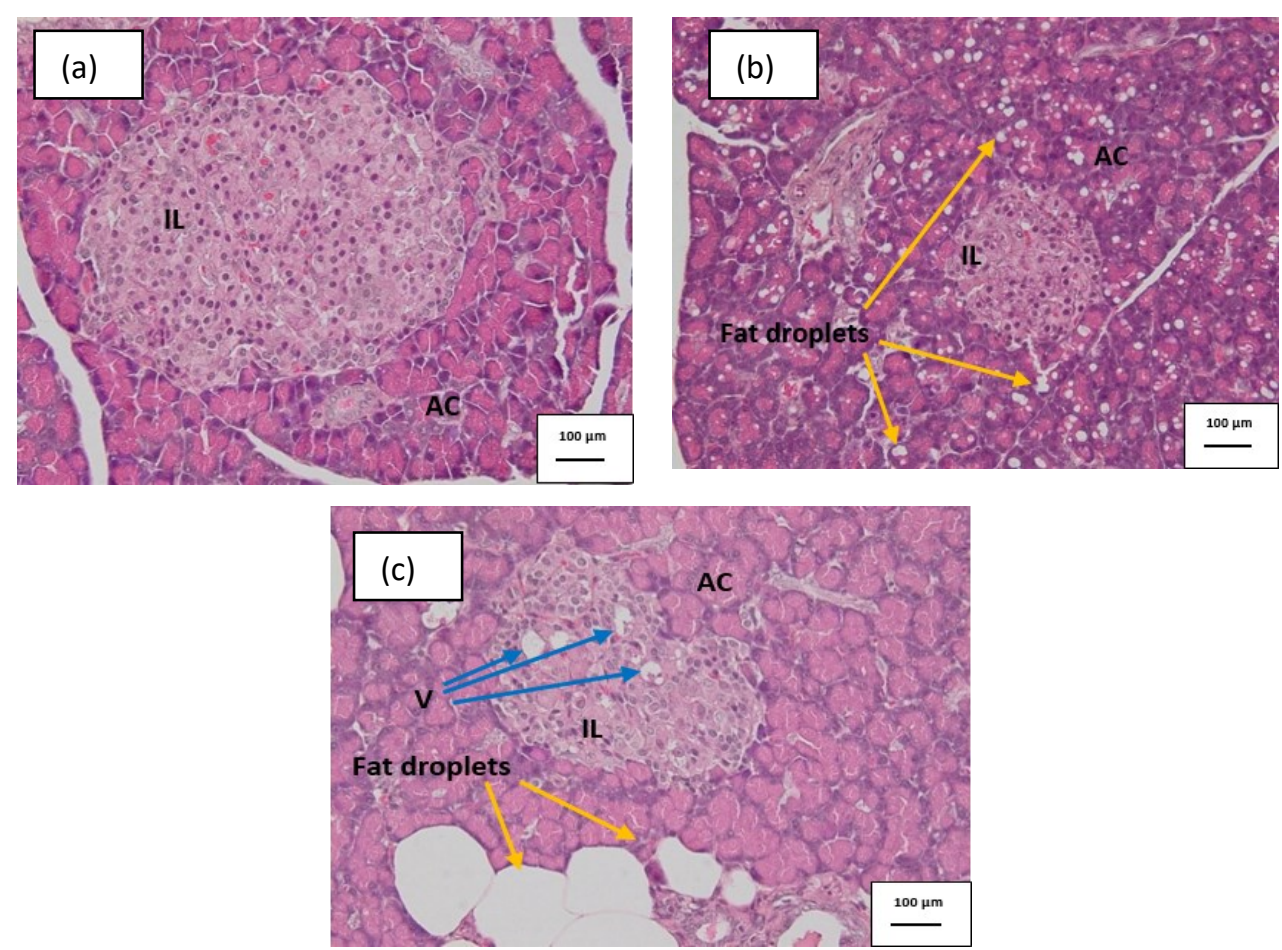

Figure 4. Light photomicrographs of the pancreatic sections from (a) Normal group showing a normal architecture of the pancreas (b) Obese rat group showing the pancreatic islet small in size with scattered fat depositions, and (c) Type 2 diabetic rat (T2DR) showing the pancreatic islet small in size, irregular in shape with the presence of scattered vacuolated cells inside. The acinar cells in the T2DR group appear to be swollen. H\&E staining (magnification $=\mathrm{X} 400)$. IL: Islet of Langerhans, AC: acini cells, V: vacuoles. 


\section{DISCUSSION}

We have successfully developed an ideal model of type 2 diabetes mellitus (T2DM) that closely resembles the metabolic characteristics and natural history of human T2DM. This study demonstrates the clinical features of T2DM, such as hyperglycaemia, decreased bodyweight, increased water intake or polydipsia, increased 24-hour urine volume, or polyuria with urine microalbuminuria and hyperlipidaemia. Therefore, a low dose of streptozotocin (STZ) at $35 \mathrm{mg} / \mathrm{kg}$ given to the obese rats following the highfat diet (HFD) feeding is capable of developing type 2 diabetic rat (T2DR). Our HFD was formulated as $31.1 \%$ of fat to produce insulin resistance after 6 weeks of feeding. Obesity is caused by environmental manipulation rather than genes, ${ }^{9}$ thus our animal model is good to model the human condition more accurately than genetic models of obesityinduced diabetes. Although fasting blood glucose levels increased significantly one week after post-induction of STZ compared to the control group, there were no significant differences in insulin levels between the groups. Because insulin is produced by beta cells in the pancreatic islets of Langerhans, this indicates that there were no significant changes in insulin production during the early stage of T2DR.

The animal model in this study was achieved by combining HFD feeding, which caused insulin resistance, and a low dose of STZ treatment, which caused the initial beta-cell dysfunction.5,25 STZ treatment following a HFD, would reduce slightly the functional capacity of the pancreatic $\beta$ cells without compromise the insulin secretion completely. It is worth noting that STZ at a low dose may only cause mild dysfunction or destruction to pancreatic cells. ${ }^{5}$ Meanwhile, at high doses, STZ has been linked to pancreatic cell destructions that can lead to necrosis. ${ }^{7}$ STZ enters the pancreatic $\beta$-cell via a glucose transporter-GLUT2 and causes alkylation of DNA. The function of the beta cells is to secrete insulin to facilitate glucose uptake into glucose recipient organs. Saturated fats, which are primarily derived from the diet are strongly associated with insulin resistance, which competes with glucose for uptake and metabolism in tissues. ${ }^{26}$

In obese rats, induction of low-dose STZ gradually diminishes the structures and functions of pancreatic $\beta$ cells, ${ }^{27}$ compromising insulin production and altering glucose homeostasis. T2DM is characterised by a progressive decline in insulin action i.e. insulin resistance, followed by beta cell inability to compensate for insulin resistance. 5 This model also produces stable and long-lasting hyperglycaemia, for example, rats fed a HFD for 5 weeks before STZ (35 mg/kg) administration, had a constant high level of blood glucose (250-300 mg/dL) for 14 weeks. ${ }^{28}$

IMJM Volume 20 No.4, October 2021
With regards to the physical changes, the T2DR demonstrated a significant weight loss compared to other groups. The reduction of body weight has been attributed to increased glycogenolysis, lipolysis, and gluconeogenesis. As diabetes progresses, the process of gluconeogenesis is required to provide glucose, which is mostly derived from fat and protein, and subsequently reducing these body stores and causing weight loss. ${ }^{29}$ Furthermore, prolonged T2DR represents a variant of features including renal changes (increased levels of blood urea nitrogen (BUN), creatinine and microalbuminuria), and liver changes (increase in liver enzymes). Exogenous compounds are metabolised and removed from the body by the kidneys and liver, so the results reflect an altered function of these organs in T2DR. ${ }^{30}$ T2DR had significantly higher total cholesterol, triglyceride, and LDL-C levels than other groups, which is consistent with previous research. ${ }^{31}$ The hypertriglyceridaemia in T2DR may be caused by increased triglycerides absorption and formation in the form of chylomicrons following exogenous consumption of a HFD or decreased triglyceride uptake in peripheral tissues. ${ }^{32}$ In diabetics, increased dietary cholesterol absorption from the small intestine following HFD feeding may result in hypercholesterolaemia. ${ }^{33} \mathrm{~A}$ significant increase in the atherogenic index (AI) in T2DR suggested that this model could be useful for studying cardiovascular disease, which is one of the diabetic complications.

In histomorphology, the pancreas from the T2DR demonstrated pathological changes in both exocrine and endocrine components. The islet of Langerhans appeared smaller and irregular, with scattered vacuolated cells inside. The morphological and functional changes are the results of a compensatory mechanism by the beta cells in response to increased insulin demand during insulin resistance. When beta-cell integrity is diminished and needs to be restored, compensation occurs. Beta-cell dysfunction is a known critical determinant for type 2 diabetes. ${ }^{34}$ A previous study that demonstrated beta-cell atrophy in hyperglycaemic weanling rats exposed to a high-fat diet during gestation supported the atrophy of the islets in this study. ${ }^{35}$ However, most previous studies demonstrated mildly enlarged beta cells as a result of hypertrophy and hyperplasia that occur during the compensatory process to increase beta-cell mass in response to hyperglycaemia. ${ }^{36,26}$ The beta cells secrete large amounts of insulin during the compensatory mechanism; however, as the disease progresses, this beta-cell function deteriorates, leading to a deterioration in glucose homeostasis and the subsequent development of impaired glucose tolerance and frank diabetes. ${ }^{37}$ Moreover, the absolute number of beta cells in the pancreatic islets of Langerhans determines the total amount of insulin released. ${ }^{38}$ Although 
the contribution of beta-cell mass and function is attributed to the pathogenesis of diabetes, the precise mechanism of its involvement remains unknown. The architectures and betacell mass regulation differ between humans and rodents, however, animal models have been widely used in diabetic studies due to the limitation of the availability of human samples and a lack of technologies to investigate human beta -cell biology. 38

\section{CONCLUSION}

In conclusion, we were able to create an animal model of type 2 diabetic rats from normal Sprague Dawley rats by giving a combination of a HFD and a low dose of STZ induction. This study shows that, besides genetic influences, carbohydrate metabolism also plays an important role in the development of Type 2 diabetes mellitus. Our animal model demonstrated the metabolic features of human T2DM, less expensive and easy to develop for diabetic research.

\section{DECLARATIONS OF INTEREST}

The authors declare no conflicts of interest.

\section{ACKNOWLEDGEMENT}

The authors would like to thank Ministry of Agriculture and Agro-based Industry (MOA) for the financial support (304.PPSK.6150169.K123).

\section{REFERENCES}

1. Punthakee Z, Goldenberg R, Katz P. Definition, classification and diagnosis of diabetes, prediabetes and metabolic syndrome. Can J Diabetes 2018;42:S10-S15.

2. Zheng Y, Ley SH, Hu FB. Global aetiology and epidemiology of type 2 diabetes mellitus and its complications. Nat Rev Endocrinol 2018;14(2):88-98. doi:10.1038/nrendo.2017.151.

3. American Diabetes Association. Classification and Diagnosis of Diabetes: Standards of Medical Care in Diabetes-2021. Diabetes Care. 2021;44(Supplement 1): S15-S33.

4. Petersen MC and Shulman GI. Mechanisms of insulin action and insulin resistance. Physiol Rev 2018;98 (4):2133-2223.

5. Srinivasan K, Viswanad B, Asrat L, et al. Combination of high-fat diet-fed and low-dose streptozotocin-treated rat: a model for type 2 diabetes and pharmacological screening. Pharmacol Res 2005;52(4):313-320.

6. Goyal R and Jialal I. Diabetes Mellitus Type 2. [Updated 2020 Nov 20]. In: StatPearls [Internet]. Treasure Island
(FL): StatPearls Publishing; Jan 2020. https:/ / www.ncbi.nlm.nih.gov/books/NBK513253/.

7. Eddouks M, Chattopadhyay D, Zeggwagh NA. Animal models as tools to investigate antidiabetic and antiinflammatory plants. Evid-based Compl Alt. 2012;2012.

8. Simmons D. The use of animal models in studying genetic disease: transgenesis and induced mutation. Nature Education. 2008;1(1):70.

9. King AJ. The use of animal models in diabetes research. British Journal of Pharmacology. 2012;166(3):877-894. https://doi.org/10.1111/j.1476-5381.2012.01911.x.

10. Kleinert M, Clemmensen C, Hofmann S, et al. Animal models of obesity and diabetes mellitus. Nat Rev Endocrinol 2018;14:140-162. https://doi.org/10.1038/ nrendo.2017.161.

11. Winzell MS and Ahrén, B. The high-fat diet-fed mouse: a model for studying mechanisms and treatment of impaired glucose tolerance and type 2 diabetes.

Diabetes. 2004;53(suppl 3):S215-S219.

12. Nor NAM, Noordin L, Abu Bakar NH, et al. Evaluation of antidiabetic activities of Etlingera elatior flower aqueous extract in vitro and in vivo. J Appl Pharm Sci 2020;10(08):043-051.

13. Lavoz C, Rodrigues-Diez RR, Plaza A, et al. VEGFR2 Blockade Improves Renal Damage in an Experimental Model of Type 2 Diabetic Nephropathy. J Clin Med 2020;9(2):302. https://doi.org/10.3390/jcm9020302.

14. Al-Awar A, Kupai K, Veszelka M, et al. Experimental diabetes mellitus in different animal models. J Diabetes Res 2016; 2016.

15. Vatandoust N, Rami F, Salehi AR, et al. Novel High-Fat Diet Formulation and Streptozotocin Treatment for Induction of Prediabetes and Type 2 Diabetes in Rats. Adv Biomed Res 2018;7:107. https://doi.org/10.4103/ abr.abr_8_17.

16. Nor NAM, Azmi NA, Noordin L, Abu Bakar NH, et al. Aqueous extract of Etlingera elatior flowers improved blood glucose control, kidney function and histology of streptozotocin-induced diabetic rat. JSSM 2019;14(3):80 91.

17. Dastan D, Salehi I, Komaki A, et al. Protective effects of the traditional herbal formulation on oxidative stress, learning and memory in the animal model of type 2 diabetes. Physiology and Pharmacology (Iran). 2020;24 (3):174-184.

18. Kowluru RA. Retinopathy in a diet-induced type 2 diabetic rat model and role of epigenetic modifications. Diabetes. 2020;69(4):689-698.

19. Lenzen $S$. The mechanisms of alloxan-and streptozotocin-induced diabetes. Diabetologia. 2008;51 (2):216-226.

20. Rason N, Ramli NS, Safuan S, et al. Histopathological alteration in organ structures of induced-obese rats fed 
with high-fat diet. Annals of Microscopy. 2016;15:38-48.

21. Novelli ELB, Diniz YS, Galhardi CM, et al.

Anthropometrical parameters and markers of obesity in rats. Lab Anim 2007;41(1):111-119.

22. Pandit R, Phadke A, Jagtap, A. Antidiabetic effect of Ficus religiosa extract in streptozotocin-induced diabetic rats. J Ethnopharmacol 2010;128(2):462-466. https:/ / doi.org/10.1016/j.jep.2010.01.025.

23. Elobeid MA and Ahmed EA. Antidiabetic efficacy of aqueous fruit extract of Amla (Emblica officinalis, gaertn) in streptozotocin-induced diabetes mellitus in male rats. Trop J Pharm Res 2015;14(5):801-806. https:// doi.org/10.4314/tjpr.v14i5.9.

24. Bo MS, Cheah WL, Lwin S, et al. Understanding the relationship between atherogenic index of plasma and cardiovascular disease risk factors among staff of an University in Malaysia. J Nutr Metab 2018;2018.

25. Zhang Y, Wang P, Xu Y, et al. Metabolomic analysis of biochemical changes in the plasma of high-fat diet and streptozotocin-induced diabetic rats after treatment with isoflavones extract of Radix Puerariae. Evid-based Compl Alt 2016;2016:4701890. http:// dx.doi.org/10.1155/2016/4701890.

26. Cerf ME. Beta cell dysfunction and insulin resistance. Front Endocrinol 2013;4:37.

27. Guo XX, Wang Y, Wang K, et al. Stability of a type 2 diabetes rat model induced by high-fat diet feeding with low-dose streptozotocin injection. J Zhejiang Univ Sci B 2018;19(7):559-569.

28. Danda RS, Habiba NM, Rincon-Choles H, et al. Kidney involvement in a nongenetic rat model of type 2 diabetes. Kidney International. 2005;68(6):2562-2571. http://dx.doi.org/10.1111/j.1523-1755.2005.00727.x.

29. Fine EJ and Feinman RD. Thermodynamics of weight loss diets. Nutr Metab 2004;1:15.

30. Imam MU, Musa SN, Azmi NH, et al. Effects of white rice, brown rice and germinated brown rice on antioxidant status of type 2 diabetic rats. Int J Mol Sci 2012;13:12952-12969. https://doi.org/10.3390/ ijms131012952.

31. Umar Imam M, Ismail M, George A, et al. Aqueous leaf extract of Clinacanthus nutans improved metabolic indices and sorbitol-related complications in type II diabetic rats (T2D). Food Sci Nutr 2019;7(4):1482-1493.

32. Srinivasan K, Patole PS, Kaul CL, et al. Reversal of glucose intolerance by pioglitazone in high-fat diet fed rats. Methods. Find. Exp. Clin. Pharmacol. 2004;26:32733.

33. Colca JR, Dailey CF, Palazuk BJ, et al. Pioglitazone hydrochloride inhibits cholesterol absorption and lowers plasma cholesterol concentrations in cholesterol fed rats. Diabetes. 1991;40:1669-74.
34. Ashcroft FM and Rorsman, P. Diabetes mellitus and the beta cell: the last ten years. Cell. 2012;148:1160-1171.

35. Cerf ME, Williams K, Chapman CS, et al. Compromised beta-cell development and beta-cell dysfunction in weanling offspring from dams maintained on a high-fat diet during gestation. Pancreas. 2007;34:347-353.

36. Jones HB, Nugent D, Jenkins R. Variation in characteristics of islets of Langerhans in insulinresistant, diabetic and non-diabetic-rat strains. Int J Clin Ex. Pathol 2010; 91(3):288-301.

37. Lebovitz HE and Banerji MA. Treatment of insulin resistance in diabetes mellitus. Eur J Pharmacol 2004;490:135-46.

38. Chen C, Cohrs CM, Stertmann J, et al. Human beta cell mass and function in diabetes: Recent advances in knowledge and technologies to understand disease pathogenesis. Mol Metab 2017;6(9):943-957. https:// doi.org/10.1016/j.molmet.2017.06.019. 